\title{
Relationship between hospital volume and short-term outcomes: a nationwide population-based study including 75,280 rectal cancer surgical procedures
}

\author{
Salvatore Pucciarelli ${ }^{1}$, Manuel Zorzi ${ }^{2}$, Nicola Gennaro ${ }^{3}$, Francesco Marchegiani $^{1}$, \\ Andrea Barina ${ }^{1}$, Massimo Rugge ${ }^{2,4}$, Matteo Zuin ${ }^{1}$, Alessandro Perin ${ }^{1}$, Isacco \\ Maretto $^{1}$, Francesca Bergamo ${ }^{5}$, Caterina Boso ${ }^{6}$, Emanuele Damiano Luca Urso ${ }^{1}$, \\ Patrick Frambach ${ }^{1}$ and Maria Chiara Corti ${ }^{3}$ \\ ${ }^{1}$ Department of Surgical, Oncological and Gastroenterological Sciences, University of Padua, Padua, Italy \\ ${ }^{2}$ Regional Health Service, Veneto Tumor Registry, Veneto Region, Padua, Italy \\ ${ }^{3}$ Regional Health Service, Epidemiology Unit, Veneto Region, Padua, Italy \\ ${ }^{4}$ Department of Medicine DIMED, Pathology and Cytopathology Unit, University of Padua, Padua, Italy \\ ${ }^{5}$ Medical Oncology 1, Veneto Institute of Oncology IOV - IRCCS, Padua, Italy \\ ${ }^{6}$ Radiation Oncology, Veneto Institute of Oncology IOV - IRCCS, Padua, Italy \\ Correspondence to: Salvatore Pucciarelli, email: puc@unipd.it \\ Keywords: rectal cancer; hospital volume; volume-outcome relationship; short-term outcomes; population study \\ Abbreviations: APR: abdominoperineal resection; Cl: confidence interval; HV: hospital volume; LOS: length of stay; OR: odds ratio \\ Received: July 12, $2017 \quad$ Accepted: February 28, $2018 \quad$ Published: March 30, 2018 \\ Copyright: Pucciarelli et al. This is an open-access article distributed under the terms of the Creative Commons Attribution License \\ 3.0 (CC BY 3.0), which permits unrestricted use, distribution, and reproduction in any medium, provided the original author and \\ source are credited.
}

\section{ABSTRACT}

There is growing interest on the potential relationship between hospital volume (HV) and outcomes as it might justify the centralization of care for rectal cancer surgery.

From the National Italian Hospital Discharge Dataset, data on 75,280 rectal cancer patients who underwent elective major surgery between 2002 and 2014 were retrieved and analyzed. HV was grouped into tertiles: low-volume performed 1-12, while high-volume hospitals performed $33+$ procedures/year. The impact of $\mathrm{HV}$ on in-hospital mortality, abdominoperineal resection (APR), 30-day readmission, and length of stay (LOS) was assessed. Risk factors were calculated using multivariate logistic regression.

The proportion of procedures performed in low-volume hospitals decreased by 6.7 percent $(p<0.001)$. The rate of in-hospital mortality, APR and 30-day readmission was $1.3 \%, 16.3 \%$, and $7.2 \%$, respectively, and the median LOS was 13 days. The adjusted risk of in-hospital mortality (OR $=1.49,95 \% \mathrm{CI}=1.25-1.78)$, APR (OR 1.10, 95\%CI 1.02-1.19), 30-day readmission (OR 1.49, 95\%CI 1.38-1.61), and prolonged LOS (OR 2.29, 95\%CI 2.05-2.55) were greater for low-volume hospitals than for high-volume hospitals.

This study shows an independent impact of HV procedures on all short-term outcome measures, justifying a policy of centralization for rectal cancer surgery, a process which is underway. 


\section{INTRODUCTION}

Rectal cancer requires complex surgical procedures and a multidisciplinary approach particularly when the tumor is located in the mid-low rectum and is locally advanced. Such complexity makes the management of this tumor similar to other cancers for which a significant association between hospital volume (HV) and outcomes has been found and centralization has been suggested $[1,2])$. Actually, previous positive experiences in some European countries [3-5] seem to support this process. However, such centralization is source of debate and robust evidence of the relationship between HV procedures and outcomes has not been clearly demonstrated yet. As randomized trials are not feasible on this topic, current evidence is mainly based on retrospective observational studies. Differences in data source, study design, patient selection, HV definition, and healthcare systems make the findings of published studies widely heterogeneous, even among systematic reviews and meta-analyses [69]. Moreover, some of the events (e.g., postoperative mortality) used to define an HV-outcome relationship occur rarely after rectal surgery. As a consequence, most studies are underpowered to capture statistically significant differences in postoperative mortality between low and high-volume hospitals. Further potential biases of previous studies derive from the use of either a single outcome or outcomes that are difficult to retrieve because they are underreported or omitted. Among the measures used to evaluate the relationship between HV and shortterm outcomes, the postoperative mortality and morbidity, the rate of abdominoperineal resection (APR), the length of stay (LOS) and the rate of unplanned readmission are the most used in the medical literature.

Given the unfeasibility of prospective randomized trial, we hypothesize that studies taking into account multiple outcome measures may strongly contribute to the debate of centralization of rectal cancer surgery. The strengths of these studies should be the longitudinal observational design and the easily retrieved data from large population-based datasets.

In this setting, we aimed to investigate whether short-term outcome measures are associated with HV after elective major rectal cancer procedures.

\section{RESULTS}

\section{Baseline patient characteristics by hospital volume}

During the study period, 75,280 patients met the inclusion criteria. (Figure 1) Low-volume hospitals performed 1-12, medium-volume 13-31, and highvolume $32+$ procedures per year. Compared with patients admitted to the high-volume, those admitted to the lowvolume hospitals were more likely to be older $(\mathrm{p}<0.001)$ male $(\mathrm{p}=0.018)$, have a worse Charlson score $(\mathrm{p}<0.001)$, have received rectal surgery in the first period of the study $(\mathrm{p}<0.001)$, have a higher rate of stoma creation $(\mathrm{p}<0.001)$, and be treated with an open approach $(p<0.001)$. Conversely, they were less likely to have been hospitalized in the year prior to the index surgery $(\mathrm{p}<0.001)$. (Table 1)

\section{Outcomes by hospital volume}

\section{In-hospital mortality}

Among the entire cohort, the overall rate of inhospital mortality was $1.3 \%$, ranging from $0.9 \%$ to $1.6 \%$ in high- and low-volume hospitals, respectively $(\mathrm{p}<0.001)$. (Table 2)

In the multivariate analysis, all the variables considered showed an independent impact on the inhospital mortality (Table 3 ). Compared with high volume hospitals, the adjusted OR of in-hospital mortality was $49 \%$ higher in low-volume hospitals. (Table 3 )

\section{Abdominoperineal resection}

Among the entire cohort, the overall rate of APR was $16.4 \%$, ranging from $14.1 \%$ to $19.4 \%$ in high- and low-volume hospitals, respectively $(\mathrm{p}<0.001)$. (Table 2 )

All the explicative variables except gender were independently associated with the APR rate. The probability of undergoing an APR was 49\% higher in patients operated on in low-volume hospitals, as compared to high-volume hospitals. (Table 3 )

\section{Length of stay}

Overall, the median (Interquartile Range) LOS was 13 (10-19) days. It was $\geq 13$ days in $39.4 \%$ and in $57.5 \%$ of cases in high- and low-volume hospitals, respectively $(\mathrm{p}=0.001)$. (Table 2)

With the exclusion of the abdominal surgery performed in the 3 years prior to the index surgery, the remaining variables were independently associated with the LOS. (Table 3)

\section{Thirty-day readmission}

Overall, the rate of 30-day readmission was $7.1 \%$, ranging from $6.8 \%$ to $7.3 \%$ in the high- and low-volume hospitals, respectively $(\mathrm{p}=0.001)$. (Table 2 )

Compared with high-volume hospitals, the adjusted OR of 30-day readmission was $10 \%$ higher in low-volume hospitals.

The HV, along with age, gender, hospitalization in the year prior to the index surgery, Charlson score, and study period was independently associated with high rates of 30-day readmission (Table 3). 


\section{Variation of hospital volume and outcomes during the study period}

Across the study period, both the proportion of patients who underwent surgery in low-volume hospitals and the proportion of hospitals that performed less than 13 procedures/year decreased, indicating an ongoing and "spontaneous" process of centralization of rectal surgery. (Figure 2) In detail, the proportion of patients treated in low-volume hospitals decreased by 6.7 percent, from $37.5 \%$ in $2002-2006$ to $30.8 \%$ in $2011-2014$, while the proportion of low-volume hospitals decreased by 6.4 percent, from $81.8 \%$ in $2002-2006$ to $75.4 \%$ in $2011-2014$.

The proportion of patients with stoma creation during index hospitalization significantly increased during the study period: $31.5 \%$ in $2002-2006,36.1 \%$ in 2007 2010 and $41.8 \%$ in $2011-2014(\mathrm{p}<0.001)$.

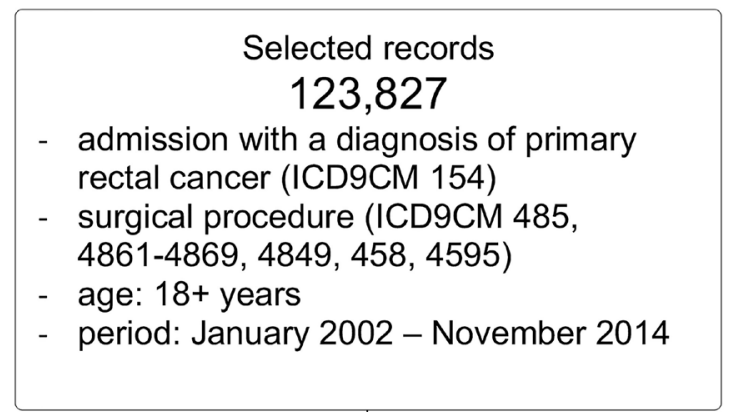

Among the four outcome measures considered, the rates of the in-hospital mortality, APR, and the LOS decreased overtime, while the rate of 30-day readmission increased. This trend was similar for each HV category. (Figure 3)

\section{DISCUSSION}

The principal aim of this study was to investigate the association between $\mathrm{HV}$ and some of the most used shortterm outcomes after major elective rectal cancer surgery. APR resection was included in the outcomes as it is widely considered to reflect the ability of the surgeon to preserve the anal sphincters and, like postoperative mortality, has been suggested to be one of top scored colorectal cancer care quality measures [10].

\section{Exclusions}

2,287 previous surgical procedure before January 1, 2002 30,615 cancer of rectosigmoid junction (ICD9CM 1541)

2,415 cancer of the anus (ICD9CM 1542, 1543)

11,257 emergent/urgent surgical procedure

1,821 previous stoma

152 discharge in acute-hospital with unavailable record

\section{Exclusion}

973 died during index hospitalization

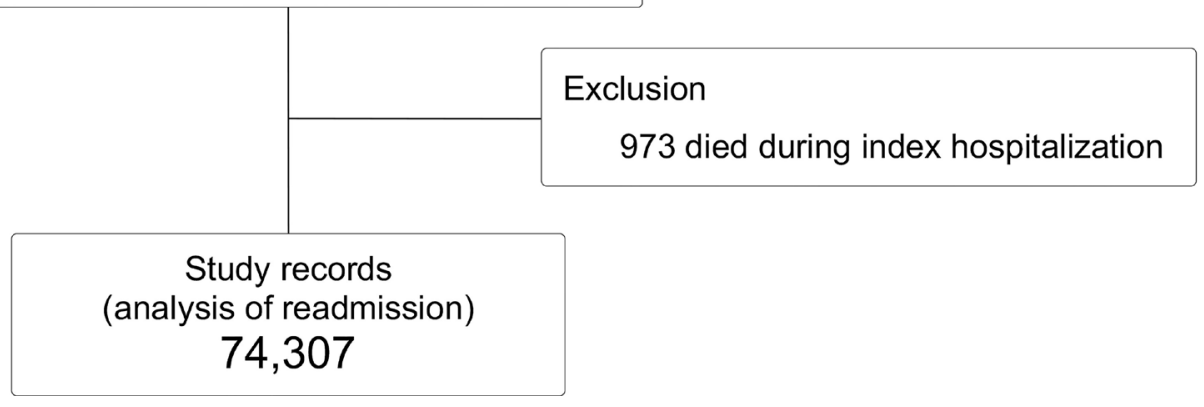

Figure 1: Flow chart of patients' selection. 
Table 1: Characteristics of patients and surgical approach by hospital volume

\begin{tabular}{|c|c|c|c|c|c|c|c|c|c|}
\hline & All patients & & $\begin{array}{l}\text { Low volum } \\
\quad(1-12)\end{array}$ & & $\begin{array}{c}\text { Medium } \\
\text { volume } \\
(13-31)\end{array}$ & & $\begin{array}{c}\text { High } \\
\text { volume } \\
(32+)\end{array}$ & & \\
\hline & $\mathbf{n}$ & column $\%$ & n & column $\%$ & $\mathbf{n}$ & column \% & $\mathbf{n}$ & column \% & p-value \\
\hline $\begin{array}{l}\text { No. of } \\
\text { Patients }\end{array}$ & 75,280 & 100.0 & 25,576 & 100.0 & 24,213 & 100.0 & 25,491 & 100.0 & \\
\hline $\begin{array}{l}\text { No. of } \\
\text { hospitals/ } \\
\text { year }\end{array}$ & 8,280 & 100.0 & 6,534 & 100.0 & 1,283 & 100.0 & 463 & 100.0 & \\
\hline \multicolumn{10}{|c|}{ Age categories } \\
\hline $18-49$ & 4,863 & $6.5 \%$ & 1,216 & $4.8 \%$ & 1,428 & $5.9 \%$ & 2,219 & $8.7 \%$ & \multirow{5}{*}{$\mathrm{p}<0.001$} \\
\hline $50-59$ & 12,323 & $16.4 \%$ & 3,643 & $14.2 \%$ & 3,823 & $15.8 \%$ & 4,857 & $19.1 \%$ & \\
\hline $60-69$ & 22,636 & $30.1 \%$ & 7,403 & $28.9 \%$ & 7,337 & $30.3 \%$ & 7,896 & $31.0 \%$ & \\
\hline $70-79$ & 25,016 & $33.2 \%$ & 9,201 & $36.0 \%$ & 8,144 & $33.6 \%$ & 7,671 & $30.1 \%$ & \\
\hline $80+$ & 10,442 & $13.9 \%$ & 4,113 & $16.1 \%$ & 3,481 & $14.4 \%$ & 2,848 & $11.2 \%$ & \\
\hline \multicolumn{10}{|l|}{ Gender } \\
\hline Male & 46,447 & $61.7 \%$ & 15,956 & $62.4 \%$ & 14,881 & $61.5 \%$ & 15,610 & $61.2 \%$ & \multirow{2}{*}{$\mathrm{p}=0.018$} \\
\hline Female & 28,833 & $38.3 \%$ & 962 & $37.6 \%$ & 9,332 & $38.5 \%$ & 9,881 & $38.8 \%$ & \\
\hline \multicolumn{10}{|c|}{ Hospitalization in the year prior to the index surgery } \\
\hline 0 & 49,883 & $66.3 \%$ & 17,685 & $69.1 \%$ & 16,256 & $67.1 \%$ & 15,942 & $62.5 \%$ & \multirow{3}{*}{$\mathrm{p}<0.001$} \\
\hline 1 & 17,308 & $23.0 \%$ & 5,651 & $22.1 \%$ & 5,480 & $22.6 \%$ & 6,177 & $24.2 \%$ & \\
\hline$>1$ & 8,089 & $10.7 \%$ & 224 & $8.8 \%$ & 2,477 & $10.2 \%$ & 3,372 & $13.2 \%$ & \\
\hline \multicolumn{10}{|c|}{ Abdominal surgery in the 3 years prior to the index surgery } \\
\hline No & 70,685 & $93.9 \%$ & 24,003 & $93.8 \%$ & 22,714 & $93.8 \%$ & 23,968 & $94.0 \%$ & \multirow{2}{*}{$\mathrm{p}=0.560$} \\
\hline Yes & 4,595 & $6.1 \%$ & 1573 & $6.2 \%$ & 1499 & $6.2 \%$ & 1,523 & $6.0 \%$ & \\
\hline \multicolumn{10}{|c|}{ Charlson score } \\
\hline 0 & 60,011 & $79.7 \%$ & 20,274 & $79.3 \%$ & 19,210 & $79.3 \%$ & 20,527 & $80.5 \%$ & \multirow{3}{*}{$\mathrm{p}<0.001$} \\
\hline $1-2$ & 13,816 & $18.4 \%$ & 4,822 & $18.9 \%$ & 4,487 & $18.5 \%$ & 4,507 & $17.7 \%$ & \\
\hline $3+$ & 1,453 & $1.9 \%$ & 480 & $1.9 \%$ & 516 & $2.1 \%$ & 457 & $1.8 \%$ & \\
\hline \multicolumn{10}{|c|}{ Year of index Hospitalization } \\
\hline $2002-2006$ & 26,987 & $35.8 \%$ & 10,127 & $39.6 \%$ & 8,269 & $34.2 \%$ & 8,591 & $33.7 \%$ & \multirow{3}{*}{$\mathrm{p}<0.001$} \\
\hline $2007-2010$ & 24,491 & $32.5 \%$ & 8,117 & $31.7 \%$ & 7,706 & $31.8 \%$ & 8,668 & $34.0 \%$ & \\
\hline $2011-2014$ & 23,802 & $31.6 \%$ & 7,332 & $28.7 \%$ & 8,238 & $34.0 \%$ & 8,232 & $32.3 \%$ & \\
\hline \multicolumn{10}{|c|}{ Stoma creation during the index hospitalization } \\
\hline Yes & 27,306 & $36.3 \%$ & 8,481 & $33.2 \%$ & 9,287 & $38.4 \%$ & 9,538 & $37.4 \%$ & \multirow{2}{*}{$\mathrm{p}<0.001$} \\
\hline No & 47,974 & $63.7 \%$ & 17,095 & $66.8 \%$ & 14,926 & $61.6 \%$ & 15,953 & $62.6 \%$ & \\
\hline \multicolumn{10}{|c|}{ Surgical approach } \\
\hline Open & 58,901 & $78.2 \%$ & 21,118 & $82.6 \%$ & 18,079 & $74.7 \%$ & 1,9704 & $77.3 \%$ & \multirow{2}{*}{$\mathrm{p}<0.001$} \\
\hline Laparoscopy & 16,379 & $21.8 \%$ & 4,458 & $17.4 \%$ & 6,134 & $25.3 \%$ & 5,787 & $22.7 \%$ & \\
\hline
\end{tabular}


Table 2: Short-term outcomes by hospital volume

\begin{tabular}{|c|c|c|c|c|c|c|c|c|c|}
\hline & All patients & & $\begin{array}{c}\text { Low } \\
\text { volume } \\
(1-12)\end{array}$ & & $\begin{array}{c}\text { Medium } \\
\text { volume } \\
(13-31)\end{array}$ & & $\begin{array}{c}\text { High } \\
\text { volume } \\
(32+)\end{array}$ & & \\
\hline & $\mathbf{n}$ & column $\%$ & $\mathbf{n}$ & column $\%$ & $\mathbf{n}$ & column $\%$ & $\mathbf{n}$ & column $\%$ & p-value \\
\hline No. of Patients & 75,280 & 100.0 & 25,576 & 100.0 & 24,213 & 100.0 & 25,491 & 100.0 & \\
\hline No. of hospitals/year & 8280 & 100.0 & 6534 & 100.0 & 1283 & 100.0 & 463 & 100.0 & \\
\hline \multicolumn{10}{|l|}{ Length of stay } \\
\hline Lower median (1-12) & 38,684 & $51.4 \%$ & 10,865 & $42.5 \%$ & 12,369 & $51.1 \%$ & 15,450 & $60.6 \%$ & \multirow{2}{*}{$\mathrm{p}<0.001$} \\
\hline Upper median $(13+)$ & 36,596 & $48.6 \%$ & 14,711 & $57.5 \%$ & 11,844 & $48.9 \%$ & 10,041 & $39.4 \%$ & \\
\hline \multicolumn{10}{|l|}{ Modality of discharge } \\
\hline Alive & 74,307 & $98.7 \%$ & 25,166 & $98.4 \%$ & 23,877 & $98.6 \%$ & 25,264 & $99.1 \%$ & \multirow{2}{*}{$\mathrm{p}<0.001$} \\
\hline Dead & 973 & $1.3 \%$ & 410 & $1.6 \%$ & 336 & $1.4 \%$ & 227 & $0.9 \%$ & \\
\hline \multicolumn{10}{|c|}{ Abdominoperineal resection } \\
\hline No & 63,005 & $83.7 \%$ & 20,624 & $80.6 \%$ & 20,479 & $84.6 \%$ & 21,902 & $85.9 \%$ & \multirow{2}{*}{$\mathrm{p}<0.001$} \\
\hline Yes & 12,275 & $16.3 \%$ & 4,952 & $19.4 \%$ & 3,734 & $15.4 \%$ & 3,589 & $14.1 \%$ & \\
\hline \multicolumn{10}{|l|}{ 30-day readmission } \\
\hline No & 68,974 & $92.8 \%$ & 23,334 & $92.7 \%$ & 22,088 & $92.5 \%$ & 23,552 & $93.2 \%$ & \multirow{2}{*}{$\mathrm{p}<0.001$} \\
\hline Yes & 5,333 & $7.2 \%$ & 1,832 & $7.3 \%$ & 1,789 & $7.5 \%$ & 1,712 & $6.8 \%$ & \\
\hline
\end{tabular}

The main finding of the study was that the risk of a worse outcome was significantly higher among patients who underwent surgery in low-volume than in those who underwent surgery in high-volume hospitals. This association was independent from the other covariates and was found for all the outcomes.

A second finding, made possible by the longitudinal design of the study and already observed by others [11], was the shift over time of patients admitted to high-volume hospitals. As, throughout the study period, no specific laws, policy recommendations or guidelines were adopted to influence such a process, it suggests that a process of centralization is "spontaneously" underway.

Comparisons between studies on this topic are challenging and many factors should be taken in account to explain differences in findings. Differences in the inclusion/exclusion criteria, definition of perioperative mortality and HV, study design, data source, study period, specificity of different healthcare systems, and cultural and geographical aspects may largely explain discrepancies between study results. A specific consideration should be reserved to postoperative mortality, which is a rare event in rectal cancer. In their large population-based study, Bilimoria et al. [2] evaluated perioperative mortality in seven common malignancies. The rates of postoperative mortality for rectal cancer were the lowest among all other malignancies $(1.9 \%$ in the highest and 3.0\% in the lowest volume-hospitals, respectively). The corresponding figures for other malignancies were respectively: $5 \%$ and $5.9 \%$ (colon), $6.1 \%$ and $10.9 \%$ (esophagus), $6.2 \%$ and $11.9 \%$ (liver), $5.5 \%$ and $6.4 \%$ (lung), $4.9 \%$ and $10.5 \%$ (pancreas), and $5.7 \%$ and $8.9 \%$ (stomach). Based on these figures, the sample size powered to find statistically significant differences in postoperative mortality after rectal cancer surgery is crucial [12]. The overall rate of in-hospital mortality in our study was $1.3 \%$, which is in the range $(0.79 \%$ to $5.2 \%)$ of that reported by others $[3,13-15]$; it was $0.9 \%$ and $1.6 \%$ in the highest and lowest HV tertile, respectively. With a such a small difference, only studies with a large sample size are able to capture a statistically significant difference. The only study to include more than 50,000 cases showed results similar to ours [2]. The rates of in-hospital mortality decreased during the study period for each HV tertile; nevertheless, like others [2, 16-19], we found that the HV still remained an independent risk factor for in-hospital mortality. Opposite findings were reported by studies based on smaller sample sizes $[4,13-$ $15,18,20,21]$.

Despite the exclusion of cancers located at the rectosigmoid junction, we found an APR rate of $16 \%$, which is lower than that reported by most of the other studies $[3,14-16,21,22]$. This is not surprising, because cultural aspects likely play a relevant role for this specific outcome, and may explain this discrepancy. Other explanations rely on the study period. Studies reporting on series of patients operated in the last century show rates of APR of more than 50\% [21-23]. In addition, the rate of sphincterpreserving procedures does not exactly reflect the rate of patients who are stoma-free. A proportion of patients, challenging to define, who underwent sphincter-preserving 
Table 3: Multivariate logistical regression analysis reporting the adjusted odds of each outcome

\begin{tabular}{|c|c|c|c|c|c|c|c|c|c|c|c|c|}
\hline \multirow[b]{2}{*}{ Variables } & \multicolumn{3}{|c|}{ In-hospital mortality } & \multicolumn{3}{|c|}{ Abdominoperineal resection } & \multicolumn{3}{|c|}{ Length of stay } & \multicolumn{3}{|c|}{ 30-day readmission } \\
\hline & OR & \multicolumn{2}{|c|}{ IC $95 \%$} & OR & \multicolumn{2}{|c|}{ IC $95 \%$} & OR & \multicolumn{2}{|c|}{ IC $95 \%$} & OR & \multicolumn{2}{|c|}{ IC $95 \%$} \\
\hline \multicolumn{13}{|c|}{ Volume (procedures/year) } \\
\hline High $(32+)$ & 1.00 & & & 1.00 & & & 1.00 & & & 1.00 & & \\
\hline Medium (13 - 31) & 1.41 & 1.17 & 1.70 & 1.16 & 1.07 & 1.26 & 1.54 & 1.37 & 1.74 & 1.09 & 1.00 & 1.18 \\
\hline Low $(1-12)$ & 1.49 & 1.25 & 1.78 & 1.49 & 1.38 & 1.61 & 2.29 & 2.05 & 2.55 & 1.10 & 1.02 & 1.19 \\
\hline \multicolumn{13}{|c|}{ Age categories (years) } \\
\hline $18-49$ & 1.00 & & & 1.00 & & & 1.00 & & & 1.00 & & \\
\hline $50-59$ & 4.93 & 1.78 & 13.62 & 1.06 & 0.95 & 1.17 & 1.05 & 0.97 & 1.13 & 0.90 & 0.79 & 1.03 \\
\hline $60-69$ & 6.53 & 2.42 & 17.67 & 1.14 & 1.04 & 1.26 & 1.23 & 1.15 & 1.33 & 0.95 & 0.84 & 1.08 \\
\hline $70-79$ & 15.31 & 5.70 & 41.08 & 1.46 & 1.32 & 1.60 & 1.70 & 1.58 & 1.83 & 1.00 & 0.88 & 1.13 \\
\hline $80+$ & 30.47 & 11.33 & 81.92 & 1.68 & 1.51 & 1.86 & 2.31 & 2.12 & 2.50 & 1.21 & 1.05 & 1.38 \\
\hline \multicolumn{13}{|l|}{ Gender } \\
\hline Male & 1.00 & & & 1.00 & & & 1.00 & & & 1.00 & & \\
\hline Female & 0.66 & 0.57 & 0.76 & 1.01 & 0.97 & 1.06 & 0.89 & 0.86 & 0.92 & 0.79 & 0.75 & 0.84 \\
\hline \multicolumn{13}{|c|}{ Hospitalization in the year prior to the index surgery } \\
\hline 0 & 1.00 & & & 1.00 & & & 1.00 & & & 1.00 & & \\
\hline 1 & 1.12 & 0.96 & 1.31 & 1.48 & 1.41 & 1.55 & 1.16 & 1.11 & 1.21 & 1.20 & 1.12 & 1.29 \\
\hline$>1$ & 1.39 & 1.15 & 1.69 & 2.00 & 1.87 & 2.13 & 1.32 & 1.25 & 1.40 & 1.27 & 1.16 & 1.40 \\
\hline \multicolumn{13}{|c|}{ Abdominal surgery in the 3 years prior to the index surgery } \\
\hline No & 1.00 & & & 1.00 & & & 1.00 & & & 1.00 & & \\
\hline Yes & 1.47 & 1.20 & 1.80 & 0.87 & 0.80 & 0.95 & 1.02 & 0.95 & 1.10 & 1.10 & 0.98 & 1.23 \\
\hline \multicolumn{13}{|l|}{ Charlson score } \\
\hline 0 & 1.00 & & & 1.00 & & & 1.00 & & & 1.00 & & \\
\hline $1-2$ & 1.43 & 1.23 & 1.67 & 0.98 & 0.92 & 1.03 & 1.27 & 1.21 & 1.33 & 1.29 & 1.20 & 1.39 \\
\hline $3+$ & 3.60 & 2.80 & 4.63 & 0.85 & 0.73 & 0.98 & 1.62 & 1.43 & 1.84 & 1.82 & 1.54 & 2.16 \\
\hline \multicolumn{13}{|c|}{ Year of index Hospitalization } \\
\hline $2002-2006$ & 1.00 & & & 1.00 & & & 1.00 & & & 1.00 & & \\
\hline $2007-2010$ & 0.80 & 0.68 & 0.94 & 0.83 & 0.78 & 0.89 & 0.61 & 0.56 & 0.66 & 1.15 & 1.06 & 1.24 \\
\hline $2011-2014$ & 0.71 & 0.60 & 0.84 & 0.85 & 0.80 & 0.91 & 0.40 & 0.37 & 0.43 & 1.34 & 1.24 & 1.45 \\
\hline \multicolumn{13}{|c|}{ Stoma creation during the index hospitalization } \\
\hline No & 1.00 & & & 1.00 & & & 1.00 & & & 1.00 & & \\
\hline Yes & 1.04 & 0.91 & 1.19 & 0.72 & 0.69 & 0.76 & 1.53 & 1.47 & 1.58 & 1.62 & 1.53 & 1.72 \\
\hline \multicolumn{13}{|l|}{ Surgical approach } \\
\hline Open & 1.00 & & & 1.00 & & & 1.00 & & & 1.00 & & \\
\hline Laparoscopy & 0.47 & 0.38 & 0.59 & 0.71 & 0.67 & 0.76 & 0.54 & 0.51 & 0.56 & 1.07 & 1.00 & 1.15 \\
\hline
\end{tabular}

surgery either had the stoma never closed or underwent a stoma creation as a consequence of an anastomotic leak. Compared to perioperative mortality, the association between HV and the rate of APR or permanent colostomy is less debated and the majority of authors report findings similar to ours $[6,9,11,15-20,23-27]$, while only a few disagree $[14,28,21]$.

We found a median LOS of 13 days which decreased overtime. While the LOS varies between studies due to differences in healthcare systems and cultural attitude [13, 
15], a decreasing LOS over time is a largely observed phenomenon as a consequence of cost-containment policies and of the improvement of available home health care and skilled facilities. Interestingly, Balentine et al.
[28] reported a greater likelihood of discharge to home for colorectal cancer patients admitted in high-volume vs. low-volume hospitals. There is large agreement on the favorable impact of $\mathrm{HV}$ on this outcome $[6,13,15,17]$.

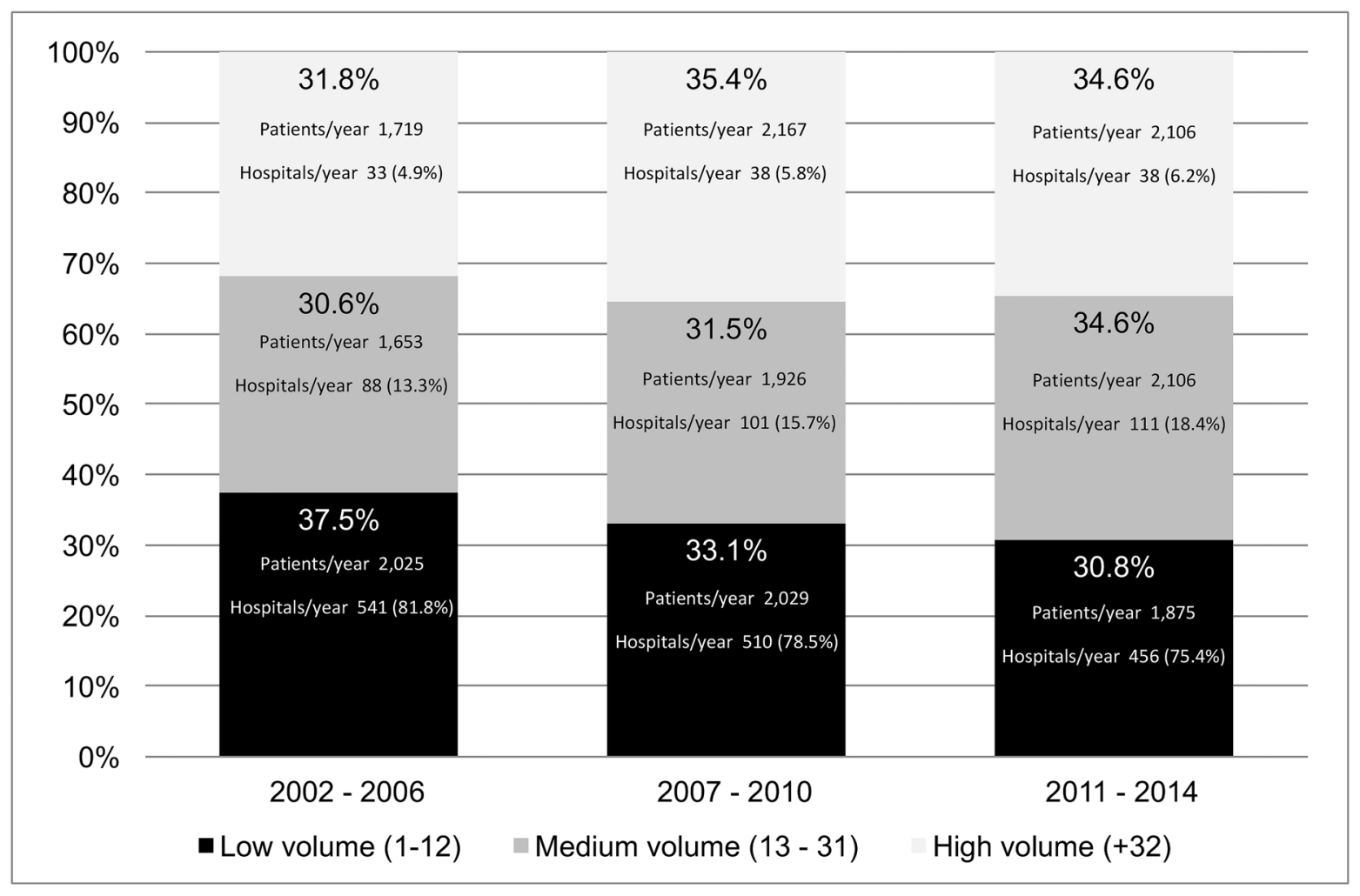

Figure 2: Distribution of patients according to the hospitals' annual volume of procedures, by period.

\section{a. In-hospital mortality}

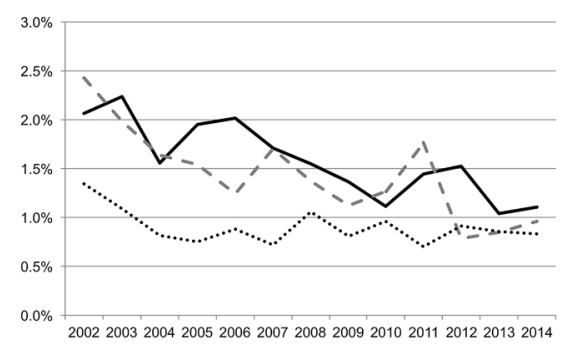

c. Length of stay

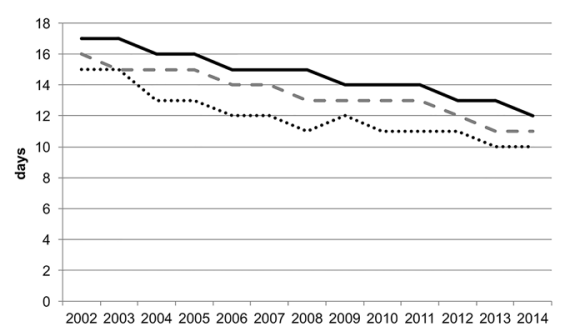

b. Abdominoperineal resection

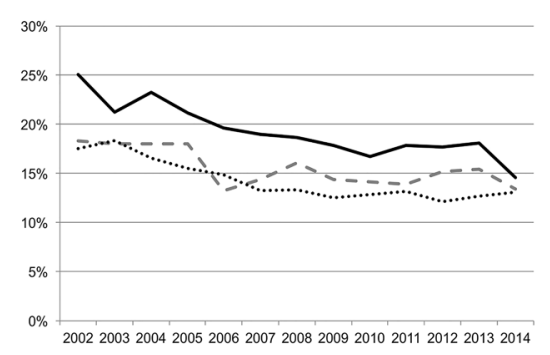

d. 30-day readmission

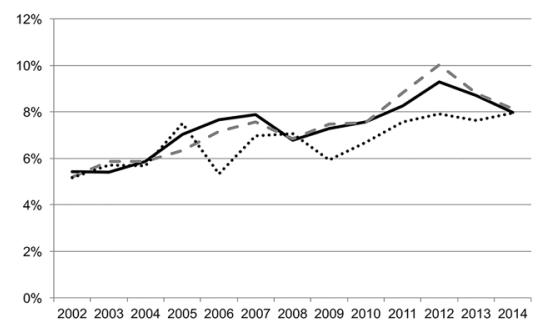

-Low volume $\quad-$ Medium volume

...High volume

Figure 3: Temporal trends of the outcome measures according to the hospitals' annual volume of procedures. 
The association of the 30-day readmission and HV is frequently reported for colorectal cancer, while few studies specifically report on this relationship after major elective rectal cancer surgery. Our finding of $7.2 \%$ of 30 day readmission compares favorably with $10.7 \%$ reported by Doumouras e al. [29] Similar to Schneider et al. [30], we found that the risk of readmission increased across the study periods. In the multivariate analysis, being operated on in more recent time periods independently increased the odds of readmission. Some temporal factors might have had an impact on this finding. Neoadjuvant therapy has been increasingly used and is currently standard of care for mid-low locally advanced rectal cancer; however, it is also considered a potential risk factor of postoperative morbidity. In addition, the proportion of patients having a covering stoma during the study period increased from $31.5 \%$ in $2002-2006$ to $41.8 \%$ in 2011 2014. As postoperative morbidity and stoma-related problems have been found to impact negatively on 30 day readmission [29, 30], it is reasonable to explain the increased rates of 30-day hospitalization with the increased use of neoadjuvant treatments and covering stoma. Furthermore, early discharge is thought to have an impact on readmissions; however, several studies found either no impact or an inverse association between LOS and the rate of 30-day readmission [29, 30].

The main strength of this study is the large sample size, the national-based source of data and the evaluation of multiple outcomes. The database does not contain any missing data which guarantees consistency of the analyses performed. Furthermore, the longitudinal design of the study produced a picture of variation over time of treatments and outcomes. Finally, unlike older studies, this analysis refers to a study period when neoadjuvant therapy, total mesorectal excision technique and the laparoscopic approach were widely diffused.

Limitations of the study are mainly related to the data source. Given its administrative nature, the data-base used does not allow for risk stratification. Clinical data such as tumor size, stage, tumor distance from the anal sphincter, or the use of adjuvant radiation or chemotherapy all of which may have an impact on short-term outcomes are not available. Likewise, the surgeon who performed the procedure is not identifiable and therefore the impact of surgeon volume on the outcomes is lacking. Although in some studies [21], the impact of surgeon volume on the outcomes has been found to be more relevant than $\mathrm{HV}$, the latter appears to be an appropriate surrogate for surgeon volume in colorectal resections [31]. A further weakness is related to the lack of reliable data on postoperative complications. We excluded this outcome because it has been demonstrated that the information and coding on postoperative complications are often omitted or underreported. [32] Moreover, we could not report on long-term outcomes (i.e., overall and disease-free survival) because a link with databases with information on the vital status of patients was not feasible.
Finally, the translation of our findings in different healthcare systems should be verified but it is possible that proposed observations are generalizable to countries with a healthcare system similar to ours.

In conclusion, we report convincing evidence that hospital volume is independently associated with shortterm outcomes in the setting of the elective complex rectal cancer surgery. These findings do support the hypothesis that a process of centralization might improve outcomes. Such a process is spontaneously underway, without the aid of policy decisions or guideline recommendations.

\section{MATERIALS AND METHODS}

\section{Study design and data source}

This was a retrospective, longitudinal, nationalbased cohort study. The data were retrieved from the administrative National Italian Hospital Discharge Dataset, which was established in 1996 and is currently utilized by the Italian Ministry of Health for administrative purposes (reimbursement of hospitals based on the Diagnosis-Related Group system). A national annual report on hospital admissions is available on-line for epidemiological studies; furthermore, the Ministry supplies researchers with anonymized data from the database [33]. Healthcare in Italy is universally delivered by public funds. The hospitals included in the national health system received the accreditation by the National Ministry of Health. This guarantees a standard in the medical treatment among them. The accreditation system has been adopted in Italy since 1992 .

For the aims of this study, Ministry of Health provided data on admissions that took place from January 1, 2000 to December 31, 2014.

The hospital discharge form reports on patient demographics, date of admission, surgical procedures and discharge. It codes for one primary and five secondary diagnoses and up to six performed procedures, surgical approach (open or laparoscopic), acuity of the admission (emergent, urgent or elective), and status at discharge (dead or alive). It does not report on stage of disease, distance of the tumor from the anal verge, preoperative or postoperative chemo and/or radiotherapy, and identification of the surgeon who performed the procedure. Moreover, information on overall and disease-free survival through linkage with other databases was not possible.

After approval of the study design by the Italian Ministry of Health, we had access to the data for this specific study. The analysis and interpretation of the data are the sole responsibility of the authors.

\section{Patient selection and definitions}

Patients were identified according to the International Classification of Diseases, Ninth Revision, Clinical Modification 2007 (ICD-9-CM). The inclusion 
criteria were: age $18+$ years, diagnosis of primary rectal cancer (ICD9-CM 154.x), major surgical procedure (ICD9-CM codes: 45.8, 45.95, 48.49, 48.5, 48.61-48.69) performed between January 2002 and November 2014. The available records regarding hospital admissions during 2000-2001 were used to exclude cases with a prevalent procedure for rectal cancer by January 1, 2002; the records regarding hospital admissions that took place in December 2014 were used to determine the 30-day readmission of patients with a hospital admission up to November 30, 2014.

The exclusion criteria were: prevalent procedure for rectal cancer before January 1, 2002, cancer of the anus (154.2-154.3) or of the recto-sigmoid junction (ICD9-CM 154.1), minor rectal cancer procedures, placement of a stoma before the index hospitalization and discharge to acute-hospitals if the record of the second hospitalization was unavailable. Patients who died during the index hospitalization were excluded from the analysis of the 30 day readmission.

\section{Outcome measures}

The outcome measures were: in-hospital mortality, which was defined as death due to any cause during the index hospitalization; rate of APR, which was the proportion of APR on the total major rectal procedures; LOS of the index hospitalization, which was defined as the difference between date of discharge and date of admission; and 30-day readmission, which was defined as any unplanned, distinct hospitalization within 30 days after the discharge of the index hospitalization.

These outcomes were chosen because they are widely used to measure the quality of healthcare and are easily retrieved from administrative databases; moreover, unlike other information such as postoperative morbidity, recording of these outcomes is mandatory and less frequently burdened by coding errors.

\section{Hospital volume and additional covariates}

Hospital volume was calculated as the average annual number of rectal cancer procedures performed at each hospital during the study period. We defined the thresholds of volume tertiles calculated on the whole study population and the hospitals were then categorized as low, medium and high volume for each study year accordingly.

The following additional covariates were assessed for the prediction of the outcomes of interest: age (subdivided into four classes: 18-59, 60-69, 7079 , and $80+$ years), gender (male, female), indexes of surgical complexity or comorbidity (non-colorectal surgery-related hospitalizations in the year prior to the index hospitalization, admissions for abdominal noncolorectal cancer-related surgery and the Charlson Index both referring to the three years prior to the index hospitalization [34], year of the index hospitalization (subdivided into three classes: 2002-2006, $2007-$ 2010, 2011-2014), creation of stoma during the index hospitalization, and open or laparoscopic approach. Of course, 30-day readmission was calculated only on those patients who were alive at discharge.

\section{Statistical analysis}

The chi-square test was used to assess differences in demographics and clinical characteristics between hospitals with different levels of annual procedure volumes (low, medium and high).

Multivariate logistical regression was used to calculate the adjusted odds ratio (OR) for each of the four study outcomes (in-hospital mortality, APR, LOS, and 30-day readmission). Multilevel regression was utilized to account for the hierarchical structure of the data (first level: patient; second level: hospital). In the multilevel analysis, the LOS was categorized into two levels, i.e., under and over the median.

Statistical significance was set at $\mathrm{p}<0.05$. Stata software was used to perform all analyses (Stata Corporation, Stata Statistical Software: Release 13.0. College Station, TX).

\section{Limitations and biases}

The study is based on administrative data. Tumor size, stage, tumor distance from the anal sphincter, adjuvant radiation or chemotherapy, surgeon who performed the procedure, postoperative complications records are not present and analyzed. The database used has no link with other databases containing clinical information. The risk of biases is related to the absence of this information.

\section{Author contributions}

Salvatore Pucciarelli participated in study conception, research design and interpretation of data, drafting, critical revision of the manuscript and team supervision.

Manuel Zorzi and Nicola Gennaro participated in study conception, research design, acquisition, statistical analysis, interpretation of data and drafting of the manuscript. They were responsible for relationship with Italian Ministry of Health.

Francesco Marchegiani, Andrea Barina, Matteo Zuin, Alessandro Perin and Patrick Frambach participated in research design, acquisition, analysis, interpretation of data, drafting and critical revision of the manuscript.

Massimo Rugge, Isacco Maretto, Francesca Bergamo, Caterina Boso and Emanuele Damiano Luca Urso participated in research design, acquisition, analysis, interpretation of data and critical revision of the manuscript. 
Maria Chiara Corti participated in research design, acquisition and statistical analysis of the data. She obtained the authorization to data access from Italian Ministry of Health. She participated in drafting and revision of the manuscript.

All the mentioned authors gave final approval of the version to be published.

\section{ACKNOWLEDGMENTS}

The Authors wish to thank Dr. Flavia Carle of the Ministry of Health - Direzione Generale della Programmazione Sanitaria, for supplying the national dataset utilized for this study.

\section{CONFLICTS OF INTEREST}

The authors declare that the research was conducted in the absence of any relationship, condition or circumstance that could present potential conflicts of interest.

\section{FUNDING}

This study was supported in part by grants from the Italian Ministry of Health (Progetti ordinari di Ricerca Finalizzata N. RF-2011-02349645 assigned to Istituto Oncologico Veneto, IOV) and from the University of Padova (Progetti di Ateneo, PRAT 2015).

\section{REFERENCES}

1. Begg CB, Cramer LD, Hoskins WJ, Brennan MF. Impact of hospital volume on operative mortality for major cancer surgery. JAMA. 1998; 280: 1747-51. https://doi. org/10.1001/jama.280.20.1747.

2. Bilimoria KY, Bentrem DJ, Feinglass JM, Stewart AK, Winchester DP, Talamonti MS, Ko CY. Directing surgical quality improvement initiatives: comparison of perioperative mortality and long-term survival for cancer surgery. J Clin Oncol. 2008; 26: 4626-33. https://doi. org/10.1200/JCO.2007.15.6356.

3. Pahlman L, Bohe M, Cedermark B, Dahlberg M, Lindmark G, Sjodahl R, Ojerskog B, Damber L, Johansson R. The Swedish rectal cancer registry. Br J Surg. 2007; 94: 128592. https://doi.org/10.1002/bjs.5679.

4. Wibe A, Eriksen MT, Syse A, Tretli S, Myrvold HE, Søreide $\mathrm{O}$, and Norwegian Rectal Cancer Group. Effect of hospital caseload on long-term outcome after standardization of rectal cancer surgery at a national level. Br J Surg. 2005; 92: 217-24. https://doi.org/10.1002/bjs.4821.

5. Manchon-Walsh P, Aliste L, Espinas JA, Prades J, Guarga A, Balart J, Biondo S, Castells A, Sanjuan X, Tabernero J, Borras JM, and Catalonian Rectal Cancer Group. Improving survival and local control in rectal cancer in Catalonia
(Spain) in the context of centralisation: A full cycle audit assessment. Eur J Surg Oncol. 2016; 42: 1873-80. https:// doi.org/10.1016/j.ejso.2016.08.009.

6. Nugent E, Neary P. Rectal cancer surgery: volume-outcome analysis. Int J Colorectal Dis. 2010; 25: 1389-96. https:// doi.org/10.1007/s00384-010-1019-1.

7. Iversen LH, Harling H, Laurberg S, Wille-Jorgensen P. Influence of caseload and surgical speciality on outcome following surgery for colorectal cancer: a review of evidence. Part 1: short-term outcome. Colorectal Dis. 2007; 9: 28-37. https://doi.org/10.1111/j.1463-1318.2006.01100.x.

8. van Gijn W, Gooiker GA, Wouters MW, Post PN, Tollenaar RA, van de Velde CJ. Volume and outcome in colorectal cancer surgery. Eur J Surg Oncol. 2010; 36: S55-63. https:// doi.org/10.1016/j.ejso.2010.06.027.

9. Archampong D, Borowski D, Wille-Jorgensen P, Iversen LH. Workload and surgeon's specialty for outcome after colorectal cancer surgery. Cochrane Database Syst Rev. 2012: CD005391.

10. Patwardhan M, Fisher DA, Mantyh CR, McCrory DC, Morse MA, Prosnitz RG, Cline K, Samsa GP. Assessing the quality of colorectal cancer care: do we have appropriate quality measures? (A systematic review of literature). J Eval Clin Pract. 2007; 13: 831-45. https://doi. org/10.1111/j.1365-2753.2006.00762.x.

11. Aquina CT, Probst CP, Becerra AZ, Iannuzzi JC, Kelly KN, Hensley BJ, Rickles AS, Noyes K, Fleming FJ, Monson JR. High volume improves outcomes: The argument for centralization of rectal cancer surgery. Surgery. 2016; 159: 736-48. https://doi.org/10.1016/j.surg.2015.09.021.

12. Birkmeyer JD, Dimick JB, Birkmeyer NJ. Measuring the quality of surgical care: structure, process, or outcomes? J Am Coll Surg. 2004; 198: 626-32. https://doi.org/10.1016/j. jamcollsurg.2003.11.017.

13. Yasunaga H, Matsuyama Y, Ohe K, and Japan Surgical Society. Volume-outcome relationship in rectal cancer surgery: a new perspective. Surg Today. 2009; 39: 663-8. https://doi.org/10.1007/s00595-008-3848-x.

14. Simunovic M, To T, Baxter N, Balshem A, Ross E, Cohen Z, McLeod R, Engstrom P, Sigurdson E. Hospital procedure volume and teaching status do not influence treatment and outcome measures of rectal cancer surgery in a large general population. J Gastrointest Surg. 2000; 4: 324-30.

15. Marusch F, Koch A, Schmidt U, Pross M, Gastinger I, Lippert H. Hospital caseload and the results achieved in patients with rectal cancer. Br J Surg. 2001; 88: 1397-402. https://doi.org/10.1046/j.0007-1323.2001.01873.x.

16. Hodgson DC, Zhang W, Zaslavsky AM, Fuchs CS, Wright WE, Ayanian JZ. Relation of hospital volume to colostomy rates and survival for patients with rectal cancer. J Natl Cancer Inst. 2003; 95: 708-16.

17. Baek JH, Alrubaie A, Guzman EA, Choi SK, Anderson C, Mills S, Carmichael J, Dagis A, Qian D, Kim J, GarciaAguilar J, Stamos MJ, Bening L, et al. The association of 
hospital volume with rectal cancer surgery outcomes. Int J Colorectal Dis. 2013; 28: 191-6. https://doi.org/10.1007/ s00384-012-1536-1.

18. Leonard D, Penninckx F, Kartheuser A, Laenen A, Van Eycken E, and PROCARE. Effect of hospital volume on quality of care and outcome after rectal cancer surgery. Br J Surg. 2014; 101: 1475-82. https://doi.org/10.1002/bjs.9624.

19. Kressner M, Bohe M, Cedermark B, Dahlberg M, Damber L, Lindmark G, Ojerskog B, Sjodahl R, Johansson R, Pahlman L. The impact of hospital volume on surgical outcome in patients with rectal cancer. Dis Colon Rectum. 2009; 52: 1542-9. https://doi.org/10.1007/ DCR.0b013e3181af58f4.

20. Harling H, Bülow S, Møller LN, Jørgensen T, and Danish Colorectal Cancer Group. Hospital volume and outcome of rectal cancer surgery in Denmark 1994-99. Colorectal Dis. 2005; 7: 90-5. https://doi. org/10.1111/j.1463-1318.2004.00751.x.

21. Schrag D, Panageas KS, Riedel E, Cramer LD, Guillem JG, Bach PB, Begg CB. Hospital and surgeon procedure volume as predictors of outcome following rectal cancer resection. Ann Surg. 2002; 236: 583-92. https://doi.org/10.1097/01. SLA.0000033036.14533.BC.

22. Holm T, Johansson H, Cedermark B, Ekelund G, Rutqvist LE. Influence of hospital- and surgeon-related factors on outcome after treatment of rectal cancer with or without preoperative radiotherapy. Br J Surg. 1997; 84:657-63. https://doi.org/10.1002/bjs.1800840520.

23. Meyerhardt JA, Tepper JE, Niedzwiecki D, Hollis DR, Schrag D, Ayanian JZ, O’Connell MJ, Weeks JC, Mayer RJ, Willett CG, MacDonald JS, Benson AB 3rd, Fuchs CS. Impact of hospital procedure volume on surgical operation and long-term outcomes in high-risk curatively resected rectal cancer: findings from the Intergroup 0114 Study. J Clin Oncol. 2004; 22: 166-74. https://doi.org/10.1200/ JCO.2004.04.172.

24. McColl RJ, McGahan CE, Cai E, Olson R, Cheung WY, Raval MJ, Phang PT, Karimuddin AA, Brown CJ. Impact of hospital volume on quality indicators for rectal cancer surgery in British Columbia, Canada. Am J Surg. 2017; 213: 388-94. https://doi.org/10.1016/j.amjsurg.2016.07.007.

25. Simons AJ, Ker R, Groshen S, Gee C, Anthone GJ, Ortega AE, Vukasin P, Ross RK, Beart RW Jr. Variations in treatment of rectal cancer: the influence of hospital type and caseload. Dis Colon Rectum. 1997; 40: 641-6.

26. Ptok H, Marusch F, Kuhn R, Gastinger I, Lippert $\mathrm{H}$. Influence of hospital volume on the frequency of abdominoperineal resection and long-term oncological outcomes in low rectal cancer. Eur J Surg Oncol. 2007; 33: 854-61.

27. Iversen LH, Harling H, Laurberg S, Wille-Jørgensen P, and Danish Colorectal Cancer Group. Influence of caseload and surgical speciality on outcome following surgery for colorectal cancer: a review of evidence. Part 2: longterm outcome. Colorectal Dis. 2007; 9: 38-46. https://doi. org/10.1111/j.1463-1318.2006.01095.x.

28. Balentine CJ, Naik AD, Robinson CN, Petersen NJ, Chen GJ, Berger DH, Anaya DA. Association of high-volume hospitals with greater likelihood of discharge to home following colorectal surgery. JAMA Surg. 2014; 149: 24451. https://doi.org/10.1001/jamasurg.2013.3838.

29. Doumouras AG, Tsao MW, Saleh F, Hong D. A populationbased comparison of 30-day readmission after surgery for colon and rectal cancer: How are they different? J Surg Oncol. 2016; 114: 354-60. https://doi.org/10.1002/ jso.24334.

30. Schneider EB, Hyder O, Brooke BS, Efron J, Cameron JL, Edil BH, Schulick RD, Choti MA, Wolfgang CL, Pawlik TM. Patient readmission and mortality after colorectal surgery for colon cancer: impact of length of stay relative to other clinical factors. J Am Coll Surg. 2012; 214:390-98. https://doi.org/10.1016/j.jamcollsurg.2011.12.025.

31. Harmon JW, Tang DG, Gordon TA, Bowman HM, Choti MA, Kaufman HS, Bender JS, Duncan MD, Magnuson TH, Lillemoe KD, Cameron JL. Hospital volume can serve as a surrogate for surgeon volume for achieving excellent outcomes in colorectal resection. Ann Surg. 1999; 230:40411. https://doi.org/10.1097/00000658-199909000-00013.

32. Romano PS, Chan BK, Schembri ME, Rainwater JA. Can administrative data be used to compare postoperative complication rates across hospitals? Med Care. 2002; 40: 856-67. https://doi.org/10.1097/01. MLR.0000027452.96163.A4.

33. Ministero della Salute. Rapporto SDO primo semestre 2015 (http://www.salute.gov.it). 2015. Available at: www.salute. gov.it/portale/temi/p2_6.jsp?id=1237\&area=ricoveriOspeda lieri\&menu=vuoto. Accessed 30 November 2016.

34. Charlson ME, Pompei P, Ales KL, MacKenzie CR. A new method of classifying prognostic comorbidity in longitudinal studies: development and validation. J Chronic Dis. 1987; 40: 373-83. 